\title{
Polysiloxaneimide Membranes I. Physical Properties
}

\author{
Juin-Yih LaI, ${ }^{* \dagger}$ Mei-Hsiu LeE, ${ }^{*}$ Shih-Hsiung Chen, ${ }^{*}$ \\ and S. S. SHYU** \\ * Department of Chemical Engineering, Chung Yuan University, \\ Chung Li, Taiwan 320, Republic of China \\ ** Department of Chemical Engineering, Central University, \\ Chung Li, Taiwan 320, Republic of China
}

(Received May 26, 1994)

\begin{abstract}
Polysiloxaneimide (PSI) membranes with siloxane moieties in the main chain were prepared by polycondensation of pyromellitic dianhydride (PMDA), 4,4'-oxydianiline (ODA) and amine-terminated poly(dimethyl siloxane) (PDMS). The effect of the PDMS content on the crystallinity, mechanical properties and thermal stability of the PSI membrane were investigated. Thermal degradation of PSI membrane was studied under nitrogen atmosphere at various heating rates. The Ozawa's method was applied for calculating kinetic parameters, such as the activation energy, the pre-exponential factor and the reaction order. The thermal stability of PSI membrane was found not to be appreciably affected by introducing PDMS, especially at a lower PDMS content. The kinetic order of degradation was determined to be unity in the nitrogen atmosphere. KEY WORDS Polysiloxaneimide Membranes / Thermal Properties / Ozawa's Method / Mechanical Properties /
\end{abstract}

In recent years, the most desirable gas separation membrane materials should have high permeability, permselectivity, thermal stability and good mechanical properties. Polyimides that are synthesized from aromatic monomers exhibit excellent temperature thermal stability and mechanical properties and are considered applicable for high temperature gas separation. Indeed, many applications already exist for this class of materials. ${ }^{1}$ Siliconcontaining polyimides ${ }^{2-4}$ have been investigated in order to improve the processability, thermo-oxidative stability, mechanical properties, impact resistance and modified surface properties. Johnson et al. ${ }^{5}$ used the pyromellitic dianhydride (PMDA) to synthesize the polysiloxaneimide (PSI) containing 5 and 10 weight percent siloxane of 720,1130 , and 2130 $\left\langle M_{n}\right\rangle$. They reported the basic structureproperty relationships but did not mention
PSI membrane application. Nagase et al. ${ }^{6}$ presented the synthesis of a poly(dimethyl siloxane) (PDMS) macromonomer with a 2(3,5-diaminophenyl) ethyl group at one end, i.e., PDMS at side chains, in gas separation and ethanol permselectivity at pervaporation. The tensile strength of PDMS membrane is very low, so that it has poor film-forming properties. This disadvantage has been overcome by putting the PDMS chain into block ${ }^{7}$ or graft $^{8}$ copolymers. In this paper, a detailed study on the copolymer membranes with different dimethylsiloxane (DMS) contents was carried out to clarify the effects of DMS content on the mechanical property, the thermal stability and the crystallinity. The thermodegradation properties of novel PSI were discussed in terms of the kinetic parameters, i.e., activation energy, pre-exponential factor and reaction order.

\footnotetext{
† To whom correspondence should be addressed.
} 


\section{EXPERIMENTAL}

\section{Materials}

High purity Pyromellitic dianhydride (PMDA) and 4,4'-oxydianiline (ODA) were obtained from Merck Co. and Chriskev Co., respectively. They were subjected to a general purification method. ${ }^{9}$ Amine-terminated poly(dimethyl siloxane) (PDMS) $\left(M_{n}=900,1680\right.$, and 3000) was obtained from Shinetsu Co. and used without any further purification. Tetrahydrofuran (THF) and 1-methyl-2-pyrrolidone (NMP) were dehydrated with $\mathrm{CaH}_{2}$ and distilled at atmospheric pressure and under reduced pressure, respectively.

\section{Preparation of Homopoly (amic acid)}

$1 \mathrm{~g}(5 \mathrm{mmol})$ of ODA was dissolved in $13 \mathrm{ml}$ THF and $6.5 \mathrm{ml}$ NMP (THF: NMP $=2: 1$ volumetric ratio). After stirring for an additional 20 minutes, $1.09 \mathrm{~g}(5 \mathrm{mmol})$ of PMDA was added to the stirring solution. The overall reaction was conducted at room temperature and allowed to stand for eight hours during which time the solution viscosity rose steadily.

\section{Preparation of Poly(siloxane amic acid)}

$0.9 \mathrm{~g}(4.5 \mathrm{mmol})$ of ODA along with $7.6 \mathrm{ml}$ THF and $3.8 \mathrm{ml} \mathrm{NMP} \mathrm{were} \mathrm{added} \mathrm{dropwise} \mathrm{to}$ $0.45 \mathrm{~g}(0.5 \mathrm{mmol})$ of amine-terminated PDMS $\left(M_{n}=900\right)$ which was dissolved in $7.6 \mathrm{ml}$ THF and $3.8 \mathrm{ml}$ NMP. Then, the solution was stirred for 20 minutes. $1.09 \mathrm{~g}(5 \mathrm{mmol})$ of PMDA was added to the stirring solution. The PMDA had a total equivalent amount of mole as ODA and amine-terminated PDMS. The resulting clear viscous solution was allowed to stand for eight hours. The content and siloxane linkage of DMS mentioned were $15.2 \mathrm{wt} \%$ and $n=10$, respectively. When the DMS content was different, the amount of solvent differed. Whereas, the volumetric ratio of THF/NMP was maintained at $2 / 1$ and the casting solution was $10 \mathrm{wt} \%$.

\section{Imidization}

A thermal procedure was employed to convert the poly(amic acid) and poly(siloxane amic acid) intermediates to the imidized final products. Curing of the poly(amic acid) and poly(siloxane amic acid) to the imide form consisted of casting on poly(tetrafluoroethylene) (PTFE) sheets to a predetermined thickness and following the heat treatment below:

1) 1 hour at room temperature in a vacuum oven.

2) 2 hours at $100^{\circ} \mathrm{C}$ in a vacuum oven.

3) 3 hours at $200^{\circ} \mathrm{C}$ in a vacuum oven.

4) 1 hour at a forced air convection oven at $300^{\circ} \mathrm{C}$.

the resulting thicknesses were in the range of $50-150 \mu \mathrm{m}$.

\section{Properties Measurements}

FTIR spectra was obtained from a Hasco FT/IR-7000. WAXD scans were generated by a Shimadzu XD-5 diffractometer operating with monochromatized copper radiation. The tensile strength measurements of membranes were carried out using the Hung Ta HT-8335A instrument. The membranes were tested by the ASTM method for their tensile strengths and elongations in the dry state. Thermal analysis was measured by DuPont Thermal Gravimetrical Analyzer Model 9900 . The heating rates were at $5,10,20$, and $40^{\circ} \mathrm{C} \mathrm{min}^{-1}$.

\section{RESULTS AND DISCUSSION}

\section{FTIR Analysis of Polyimide and Polysilox- aneimide}

FTIR analysis was made to determine the degree of imidization of the prepared membranes. As shown in Figure 1 (a), the infrared spectra of the polyimide shows characteristic polyimide absorption bands near 725,1725 , and $1777 \mathrm{~cm}^{-1}$. Any presence of unimidized poly(amic acid) would be detected by a very strong absorption at approximately $1660 \mathrm{~cm}^{-1}$ and weaker absorption bands at $3200 \mathrm{~cm}^{-1}$ (OH stretch of the carboxylic acid) and at 


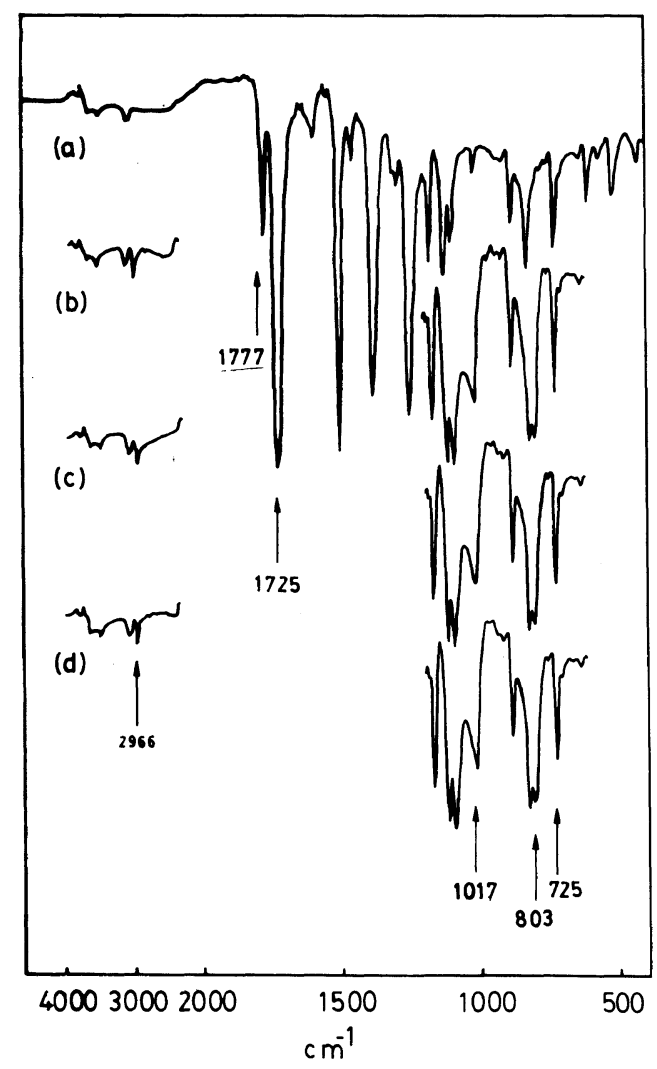

Figure 1. FTIR spectra: (a) polyimide; PSI with DMS content of $15.2 \mathrm{wt} \%$; (b) siloxane linkage of $n=10$; (c) siloxane linkage of $n=21$; (d) siloxane linkage of $n=40$.

$3350 \mathrm{~cm}^{-1}$ (NH stretch of the amide group). None of these bands were detected in the infrared spectra of the imidized membranes. The completed imidization was verified for these membranes. Figures 1 (b), (c), and (d) show the spectra of polysiloxaneimide (PSI) with DMS siloxane linkage of 10,21 , and 40 , respectively. The PSI membranes show a sharp peak at $2966 \mathrm{~cm}^{-1}$ which is due to the $\mathrm{CH}_{3}$ stretching in the amine-terminated PDMS which is not found in the FTIR spectra of the polyimide membrane. The absorption of $\mathrm{Si}-\mathrm{O}$, $\mathrm{Si}-\mathrm{C}$ are confirmed at 1017 and $803 \mathrm{~cm}^{-1}$, respectively. Thus, the existence of DMS in PSI membranes were confirmed.

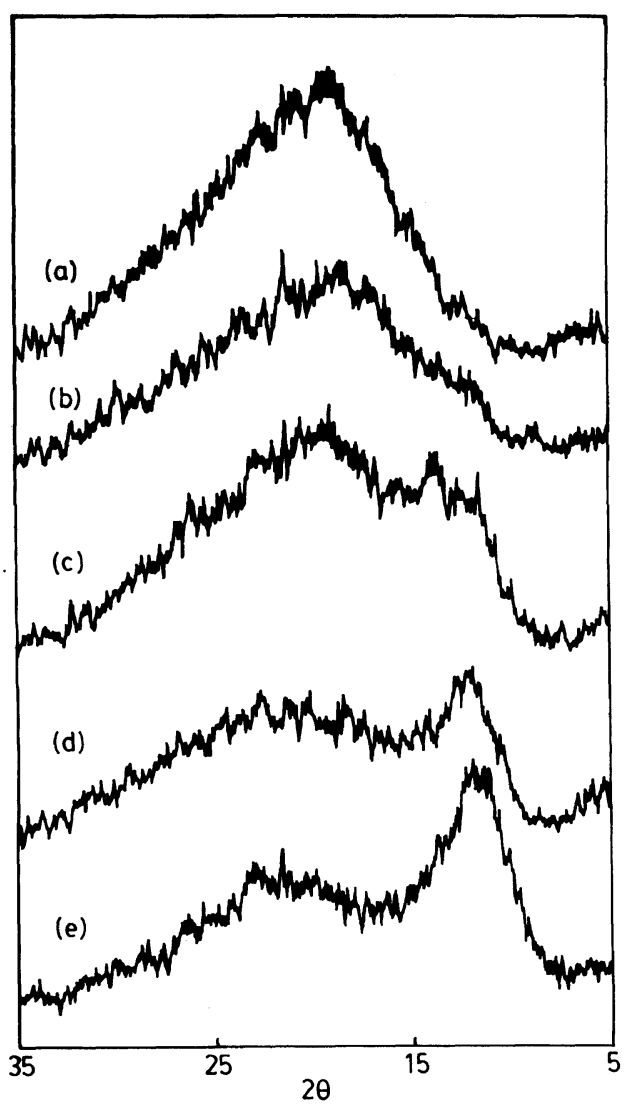

Figure 2. X-ray diffraction patterns of the polyimide and polysiloxaneimide $(n=40)$ membranes. (a) polyimide; (b) DMS content $15.2 \mathrm{wt} \%$; (c) DMS content $35.4 \mathrm{wt} \%$; (d) DMS content $53.0 \mathrm{wt} \%$; (e) DMS content $92.0 \mathrm{wt} \%$ (without ODA).

\section{$X$-ray Diffraction Studies}

Wide-angle X-ray diffraction (WAXD) studies were performed on these samples with the incident beam normal to the surface of the membranes. The diffraction pattern for the polyimide is depicted in Figure 2 (a). The pattern is similar in nature and shape to those reported for polyimide by Russell. ${ }^{10}$ The broad peak centered at $2 \theta=19.2^{\circ}$ was attributed to polymer interchain distance. The d-spacings can be calculated by applying the scattering angles $(2 \theta)$ of the peaks into the Bragg's equation, ${ }^{11} n \lambda=2 d \sin \theta$. The d-spacings determined in this manner is a measure of inter- 
chain distance of polymeric molecules. ${ }^{1}$ The PSI with lower DMS content of $15.2 \mathrm{wt} \%$, no matter the siloxane linkage number of DMS, exhibited similar diffraction patterns to that for polyimide in Figure 2 (a). The Bragg spacings (d-spacings) obtained from the WAXD data for the polyimide and PSI membranes with various siloxane linkage are 4.6 and 4.7-4.9 $\AA$, respectively. The above results suggest that the different siloxane linkage affect the interchain distance of polymer.

Figure 2 shows the effect of DMS content of the PSI on the WAXD patterns. The PSI with siloxane linkage $n=40$ has no clear crystal peak until the DMS content was up to $35.4 \mathrm{wt} \%$. An increase in the DMS content of the PSI results in an increase in the degree of the crystallization and decrease in the amorphous region. In addition, the PSI with different siloxane linkage of DMS have similar results. These phenomena might be explained as follows: The mean interchain distance of the polyimide are lower than that of PSI because the DMS can act as "spacer" in the PSI structure backbone. The induction of flexible DMS to polyimide structure increased the mean interchain distance in PSI. In other word, the DMS segment in the PSI are flexible and easy arrange to form the regular structure. The regular arrangement of DMS in the PSI increased the crystallinity with increasing DMS content. The similar phenomena were reported by Li et al. ${ }^{12}$

Influence of the DMS Content on the Mechanical Properties

Table I shows the mechanical properties of the PSI polymer could be affected by the DMS siloxane linkage and the overall DMS content. The elongations increased and the tensile strength decreased with increasing the DMS content. This can be attributed to the fact that DMS groups are flexible to promote the elongations and to degenerate the tensile strength. However, in the same DMS content, the shortest siloxane linkage $(n=10)$ of DMS
Table I. Mechanical properties of polysiloxaneimides

\begin{tabular}{|c|c|c|c|}
\hline DMS content & DMS & $\begin{array}{c}\text { Tesile } \\
\text { strength }\end{array}$ & Elongation \\
\hline$w t \%$ & linkage & $\mathrm{MPa}$ & $\%$ \\
\hline 0 & 0 & 100.1 & 12.1 \\
\hline 15.2 & 10 & 63.7 & 34.3 \\
\hline 15.2 & 21 & 65.1 & 29.0 \\
\hline 15.2 & 40 & 68.4 & 17.2 \\
\hline 26.5 & 10 & 48.6 & 54.3 \\
\hline 26.5 & 21 & 57.8 & 40.5 \\
\hline 26.5 & 40 & 61.3 & 33.3 \\
\hline 35.4 & 10 & 31.3 & 73.8 \\
\hline 35.4 & 21 & 44.9 & 61.6 \\
\hline 35.4 & 40 & 52.0 & 40.5 \\
\hline 53.0 & 10 & 12.0 & 109.9 \\
\hline 53.0 & 21 & 21.2 & 66.0 \\
\hline 53.0 & 40 & 21.9 & 51.8 \\
\hline
\end{tabular}

in PSI has the highest elongation and the lowest tensile strength. In other words, the number of siloxane linkage of DMS affects significantly the mechanical properties of PSI. It might be due to the fact that the mechanical properties of the PSI matrix with the shortest siloxane linkage possess relatively lower ODA concentration in the same DMS content. As a result, PSI with shortest siloxane linkage $(n=10)$ significantly affected in modifying PSI polymer matrix.

\section{Influence of the DMS Content on the Thermal Stability}

Figure 3 shows the thermogravimetric curves (TG) of polyimide and PSI membranes with a heating rate of $20^{\circ} \mathrm{C} \mathrm{min}^{-1}$ and a nitrogen flow rate of $100 \mathrm{ml} \mathrm{min}^{-1}$. Thermal stability of copolymer varied with levels of DMS incorporation. The initial decomposition temperature of PSI decreased with increasing DMS content. From the data of weight loss at $800^{\circ} \mathrm{C}$, samples with more DMS content had less polymer residues. This phenomenon was also observed in copolyimide ${ }^{13}$ and PI/PDMS copolymer. ${ }^{6}$ The derivative TG curves of PSI copolymers with various DMS content show 


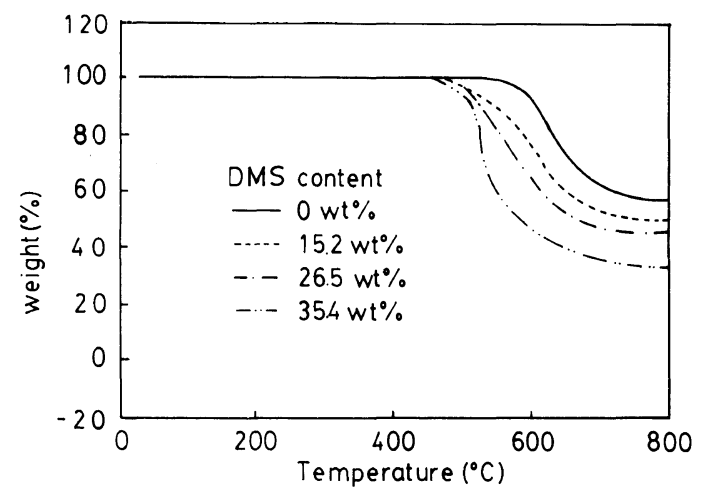

Figure 3. TG curves of polyimide and PSI with DMS siloxane linkage of $n=10$ at heating rate $20^{\circ} \mathrm{C} \mathrm{min}^{-1}$ in $\mathrm{N}_{2}$ atmosphere.

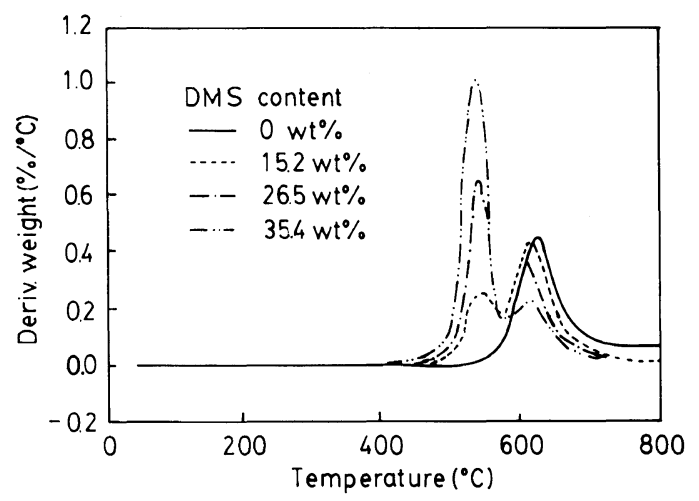

Figure 4. The derivative TG curves of polyimide and PSI with DMS siloxane linkage of $n=10$ at heating rate $20^{\circ} \mathrm{C} \mathrm{min}^{-1}$ in $\mathrm{N}_{2}$ atmosphere (data taken from Figure 3).

two maximum rate of decomposition temperature $\left(T_{\max }\right)$ as shown in Figure 4 . The first stage was subjected to the rapid decomposition of DMS while the secondary stage was due to the degradation of ODA. ${ }^{13}$ The above results might be due to the fact that the dissociation energy of $\mathrm{Si}-\mathrm{C}$ bonding is lower ${ }^{14}$ and the thermal degradation begins at the aliphatic $n$-propyl segments linking the DMS oligomers to the polyimide matrix. ${ }^{9}$ Accordingly, it is evident that the weight loss of the first stage decomposition temperature of the PSI increased with increasing the DMS content and the secondary stage decomposition temperature decreased. In the thermal stability studies,

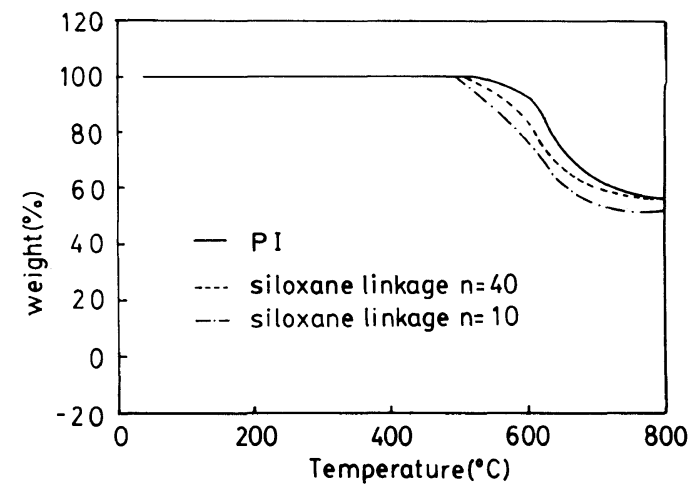

Figure 5. TG curves of polyimide and PSI with DMS content of $15.2 \mathrm{wt} \%$ at heating rate $20^{\circ} \mathrm{C} \mathrm{min}^{-1}$ in $\mathrm{N}_{2}$ atmosphere.

it is concluded that the lower DMS moiety content in PSI possesses the higher thermal stability. Nevertheless, the PSI membranes still have higher thermal stability than that of the other polymers.

The effect of the different DMS siloxane linkage with constant total DMS content of $15.2 \mathrm{wt} \%$ on the TG curves for the thermal degradation of PSI membranes are shown in Figure 5. The thermal stability varies with different siloxane linkage of DMS. From this observation, one may conclude that the longer DMS siloxanes linkage possesses the better thermal stability. The result may be due to the fact that thermal degradation begins at the aliphatic $n$-propyl segments linking the DMS oligomers to the polyimide matrix. ${ }^{9}$ Additionally, the structure of PMDA-ODA segment is considered to have an excellent thermal stability. Thus, as the DMS siloxane linkage was longer, the concentration of the $n$-propyl linkages decreased and the concentration of ODA increased in PSI copolymers backbone at the same DMS content. Moreover, from the derivative TG curves of PSI copolymer with various siloxanes linkage show two decomposition temperature except the pure polyimide, as shown in Figure 6. The behaviors of thermodegradation of PSI are quite different from the polyimide homopolymer.

In order to probe deeply into thermal deg- 


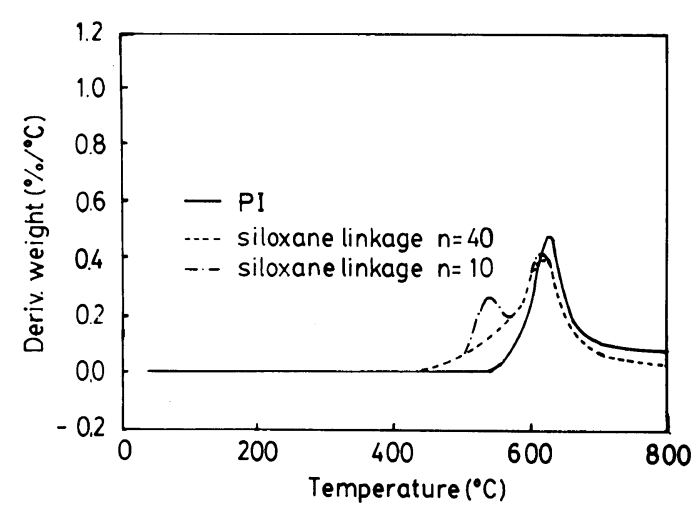

Figure 6. The derivative TG curves of polyimide and PSI with DMS content of $15.2 \mathrm{wt} \%$ at heating rate $20^{\circ} \mathrm{C} \mathrm{min}^{-1}$ in $\mathrm{N}_{2}$ atmosphere (data taken from Figure 5).

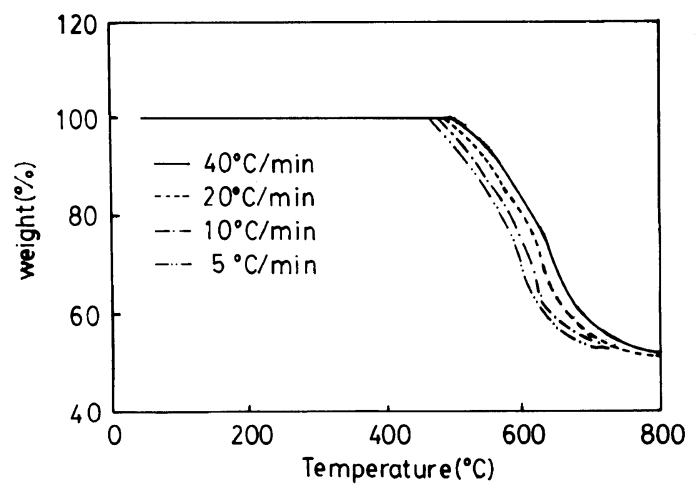

Figure 7. TG curves of PSI with DMS content of $15.2 \mathrm{wt} \%$ and DMS siloxane linkage of $n=10$ at various heating rates in $\mathrm{N}_{2}$ atmosphere.

radation of PSI, Ozawa's ${ }^{15-17}$ method was applied to calculate kinetic parameters, such as the activation energy, the pre-exponential factor and the reaction order. The results of the thermogravimetry at various heating rates in nitrogen atmosphere were plotted against the temperature in Figure 7. The derivative TG curves in nitrogen were shown in Figure 8. The activation energy was determined by Ozawa's method for a given value of weight fraction $(w t \%)$. He reported that the weight decreases to a given fraction at the temperature $T_{1}$, for the heating rate of $\beta_{1}$, at $T_{2}$ for $\beta_{2}$, and so on. The following equation could be derived.

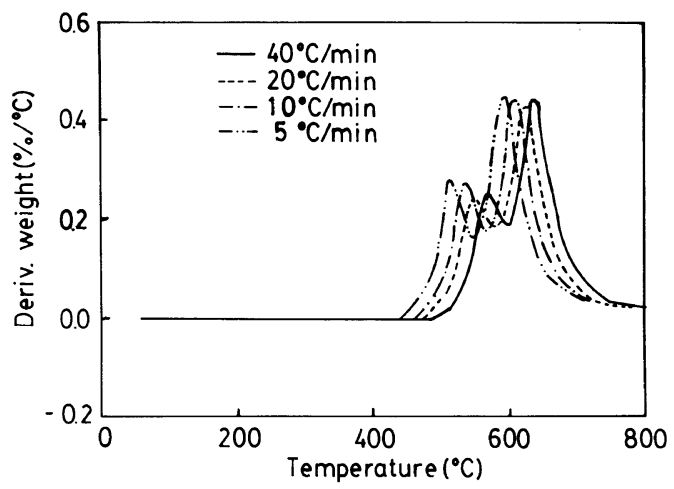

Figure 8. The derivative TG curves of PSI with DMS content of $15.2 \mathrm{wt} \%$ and DMS siloxane linkage of $n=10$ at various heating rates in $\mathrm{N}_{2}$ atmosphere (data taken from Figure 7).

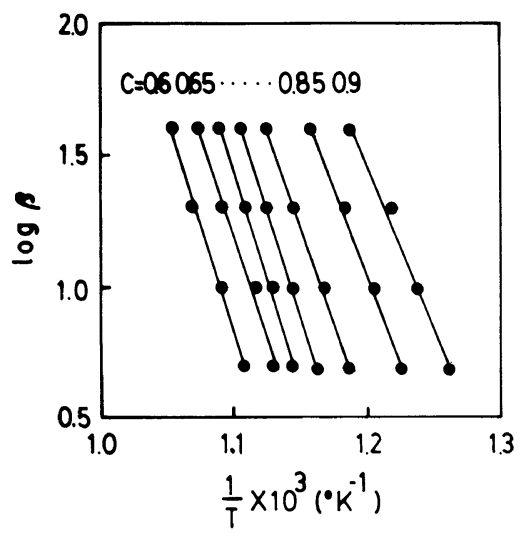

Figure 9. The plots of logarithms of heating rate $v s$. the reciprocal absolute temperature for indicated conversion of the degradation of PSI with DMS content of $15.2 \mathrm{wt} \%$ and DMS siloxane linkage of $n=10$ in the $\mathrm{N}_{2}$ atmosphere.

$$
\begin{aligned}
& -\log \beta_{1}-0.4567-\Delta E / R T_{1} \\
& \quad=-\log \beta_{2}-0.4567-\Delta E / R T_{2}=\cdots
\end{aligned}
$$

where $E$ is the activation energy and $R$ is the ideal gas constant. From Figure 7, the logarithms of the heating rates could be plotted against the reciprocal absolute temperature, until reached the given decreased weight fraction of the sample. The results are shown in Figure 9. Straight lines are drawn by the method of the least squares. From these lines the activation energies are determined as listed in Table II in nitrogen atmosphere. 
Table II. Activation energies and pre-exponential factors of the degradation of Polysiloxaneimide (with DMS $15.2 \mathrm{wt} \%$ and DMS siloxane linkage $n=10$ ) in nitrogen atmosphere

\begin{tabular}{|c|c|c|c|c|}
\hline \multirow{3}{*}{ Condition } & \multicolumn{2}{|c|}{ First stage } & \multicolumn{2}{|c|}{ Second stage } \\
\hline & $\Delta E$ & & $\Delta E$ & \\
\hline & $\mathrm{kJ} \mathrm{mol}^{-1}$ & & $\mathrm{~kJ} \mathrm{~mol}^{-1}$ & \\
\hline In $\mathrm{N}_{2}$ & 234.8 & 16.25 & 313.5 & 18.55 \\
\hline
\end{tabular}

a $A$ was calculated by Ozawa's method, $\min ^{-1}$

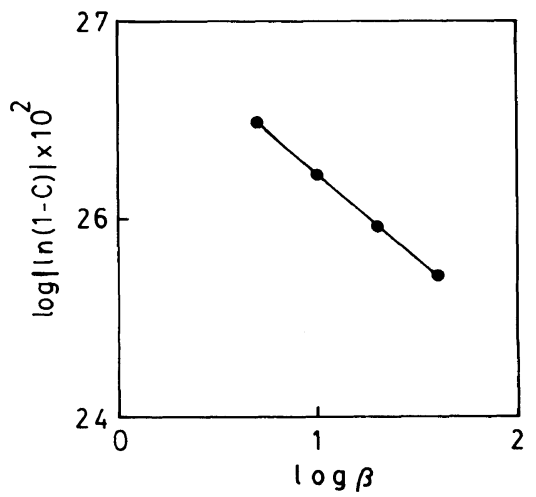

Figure 10. Relationship obtained by Ozawa's method for $n=1$ in $\mathrm{N}_{2}$ atmosphere; $\beta$, heating rate; $C$, conversion.

When kinetic order is unity, the following formula could be derived:

$$
\begin{aligned}
& \log |\ln (1-C)| \\
& \quad=\log (A E / \beta R)-2.315-0.4567 E / R T(n=1)
\end{aligned}
$$

where $A$ is the pre-exponential factor. The conversion $C$ is defined by

$$
C=1-\frac{W}{W_{0}}
$$

where $W_{0}$ and $W$ represent initial weight and weight at any time, respectively, and $n$ is the reaction order. The logarithm of $\ln (1-C)$ can be plotted against the logarithm of heating rate $(\beta)$ in nitrogen as shown in Figure 10. A good linear relationship was obtained in nitrogen. These results mean that the kinetic order is unity in nitrogen atmosphere.

\section{CONCLUSION}

The DMS moiety plays an important role in the PSI and hence the properties of the PSI significantly different to the polyimide. The mechanical properties and thermal stability of the copolymer with shorter siloxane linkage has higher influence on the polymer matrix than that of the longer siloxane linkage. The derivative TG curves of the thermogravimetric data revealed two maximum rate of decomposition temperatures that were closely related to the composition of the polymer. The kinetic order of degradation is determined to be unity in nitrogen atmosphere. Adding the DMS moiety results in an increase in the mean interchain distance, i.e., an increase in the distance between neighboring polymer chains. The results show that the thermodegradation of such polymer occurs above $400^{\circ} \mathrm{C}$ in two stages in a nitrogen atmosphere. This study shows that the prepared PSI membrane is suitable for high temperature gas separation. The gas separation performance of PSI membrane will be reported in the near future.

Acknowledgements. The authors wish to thank the National Science Council of the Republic of China for financial support (NSC83-0405-E033-001).

\section{REFERENCES}

1. K. L. Mittal, Ed., "Polyimides: synthesis, characterization, and applications," Vol. 1 and 2. Plenum Press, New York, 1984.

2. V. H. Kuckertz, Makromol. Chem., 98, 101 (1966).

3. G. C. Tesoro, G. P. Rajendran, D. R. Uhlmann, and C. E. Park, Ind. Eng. Chem. Res., 26, 1672 (1987).

4. Y. D. Moon and Y. M. Lee, J. Appl. Polym. Sci., 50, 1461 (1993).

5. B. C. Johnson, I. Yilgor, and J. E. McGrath, Polym. Prepr., 25(2), 54 (1984).

6. Y. Nagase, S. Mori, M. Egawa, and K. Matsui, Makromol. Chem., 19, 2413 (1990).

7. W. J. Ward, III, W. R. Browall, and R. M. Salemma, J. Membrane Sci., 1, 99 (1976).

8. Y. Kawakami, H. Karasawa, T. Aoki, Y. Yamamura, H. Hisada, and Y. Yamashita, Polym. J., 17(11), 1159 


\section{Polysiloxaneimide Membranes. I. Physical Properties}

(1985)

9. R. H. Bott, J. D. Summers, C. A. Arnold, L. T. Taylor, T. C. Ward, and J. E. Mcgrath, J. Adhesion, 23, 67 (1987).

10. T. P. Russell, J. Polym. Sci., B, Polym. Phys. Ed., 22, 1105 (1984).

11. L. H. Schwarts and J. B. Cohen, "Diffraction from Materials, Academic Press, New York, N.Y., 1977, Chapter 3.

12. W. Li and B. Huang, J. Polym. Sci., B, Polym. Phys.,
30, 727 (1992).

13. Y. D. Lee, C. C. Luo, and H. R. Lee, J. Appl. Polym. Sci., 41, 877 (1988).

14. N. Tsutsumi, A. Tsuji, C. Horie, and T. Kiyotsukuri, Eur. Polym. J., 24(9), 837 (1988).

15. T. Ozawa, Bull. J. Chem. Soc. Jpn., 38, 1881 (1965).

16. H. Nishizaki, K. Yoshida, and J. H. Wang, J. Appl. Polym. Sci., 25, 2869 (1980).

17. D. J. Liaw, M. C. Lin, and K. R. Lee, J. Chim. Inst. Chem. Eng., 13(3), 241 (1990). 